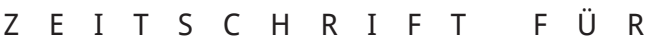

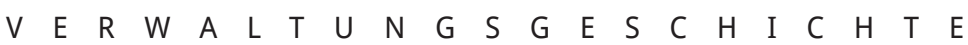

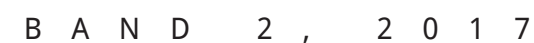

$S$ E I T

D O I : 10.2478/ADHI-2018-0027

\title{
"Les Maires en France du Consulat à nos jours" im Spiegel der aktuellen Geschichtsforschung
}

\author{
BETTINA SEVERIN-BARBOUTIE
}

Frankreich ist bis heute für seine Vielzahl eigenständiger Kommunen bekannt. Bis in das 21. Jahrhundert hinein zählte der französische Staat nie weniger als 36.000 lokale Verwaltungsbezirke. Durch die jüngste Territorialreform ist die Zahl der Kommunen zwar um mehrere Hundert zurückgegangen. "La France ne compte plus que 35.498 communes«, verkündete der Journalist Patrick Roger in Le Monde im Januar 2017. ${ }^{1}$ Doch bleibt die französische Republik bis in die Gegenwart hinein ein Staat mit einer besonders kleinteiligen lokalen Verwaltungsgeografie bei einem gleichzeitig ausgeprägten Zentralismus.

Historisch wurzelt diese lokale Verwaltungsgeografie in der Gebietsreform, die die Neuordnung der Verwaltung in der Zeit der Französischen Revolution begleitete und in deren Folge Frankreich im Dezember 1789 eine neue Territorialordnung enthielt. ${ }^{2}$ Unterste Stufe beziehungsweise kleinste territoriale Einheiten dieser neuen Ordnung waren die Munizipalitäten, die, da nicht immer neue Grenzziehungen vorgenommen wurden, häufig lokalen Verwaltungseinheiten des Ancien Régime entsprachen. In der lokalen Verwaltungsgeografie Frankreichs blieb daher die territoriale Gliederung der französischen Monarchie im 18. Jahrhundert erkennbar - zumindest teilweise. Und im Grunde gilt dieser Befund bis heute.
Jede Kommune besitzt bis heute ihre eigene Verwaltung. Sie besteht zum einen aus einem gewählten Gremium: dem Munizipalrat, zum anderen aus einem einzelverantwortlichen Verwaltungsleiter: dem Maire. Bis 1882 wurde dieser ernannt, sieht man von Unterbrechungen in politischen Umbruchzeiten ab, wobei die Nomination je nach Größe der Kommune und Staatsform entweder durch den Monarchen oder durch hohe Staatsbeamte erfolgte. Seit 1882 wird der Maire vom Munizipalrat gewählt. ${ }^{3}$

Innerhalb der Verwaltungsordnung besitzt der Maire seit jeher eine Sonderstellung. Sie ergibt sich zumal aus der Ehrenamtlichkeit seiner Tätigkeit, noch mehr aber aus der Tatsache, dass er im Unterschied zu Unterpräfekten und Präfekten, den Amtsleitern der die Kommunen überwölbenden Arrondissements und Departements, Verwalter des Staates vor Ort und Repräsentant der Mitglieder seiner Kommune gegenüber dem Gesamtstaat zugleich ist, also eine Doppelfunktion erfüllt. Der Repräsentationsanspruch, den er in diesem Zusammenhang erheben kann, hängt dabei von der Größe der Kommune ab, die er leitet und vertritt. ${ }^{4}$

In der französischen Geschichtsforschung wurden zunächst vor allem einzelne Kommunen genauer untersucht. 
Im Zuge des wachsenden Interesses der Geschichtswissenschaft an sozialgeschichtlichen Fragen in der zweiten Hälfte des 20. Jahrhunderts im Allgemeinen und an sozialen Gruppen im Besonderen ${ }^{5}$ gelangten mehr und mehr auch die kommunalen Amtsleiter und ihr sozioökonomisches beziehungsweise politisches Profil in den Blick. ${ }^{6}$ In den 1980er Jahren lancierte die Forschergruppe für die Geschichte des 19. Jahrhunderts, die der Historiker Louis Girard in Paris ins Leben gerufen hatte, ein Projekt, in welchem die Maires erstmals systematisch und im historischen Längsschnitt erforscht wurden. Im Mittelpunkt stand dabei die Frage nach ihrer Rekrutierung. Für die Forschergruppe handelte es sich nicht um die erste Studie dieser Art. Tatsächlich hatte sie in den Jahrzehnten zuvor bereits zwei vergleichbare sozialhistorische Projekte durchgeführt: in den 1960er Jahren über die Deputierten von 1837, eine Dekade später über die Generalräte von $1870 .^{7}$

Für die mehr als zwanzig Personen umfassende Forschergruppe erwies sich die sozialhistorische Längsschnittuntersuchung der Maires freilich als ungleich schwieriger als die Vorhaben, die sie in den Jahren zuvor durchgeführt hatte - zum einen wegen der Vielzahl an Kommunen, zum anderen wegen unzureichender beziehungsweise asymmetrischer Quellen. Eine zeitlich durchgehende, räumlich flächendeckende Untersuchung war daher nicht möglich, auch deshalb nicht, weil es keine einschlägigen Vorarbeiten gab. Die Gruppe entschied sich deshalb für eine räumlich wie zeitlich begrenzte Stichprobenerhebung. Räumlich konzentrierte sie sich auf die Kommunen von 13 Arrondissements (Béthune, Blois, Brignoles, Commercy, Die, Etampes, La Châtre, Le Mans, Mâcon, Saint-Denis, Sceaux, Villefranche-sur-Saône, Yssingeaux), rund 1250 an der Zahl. Zeitlich fokussierte sie die Jahre 1811, 1824, 1832, 1841, 1850, 1882, 1913, 1926 , 1936, 1954 und 1966. Ausschlaggebend für die Wahl der 13 Arrondissements waren persönliche Kriterien. Untersucht wurden Arrondissements, in denen das Team Personen kannte, die die Erhebung durchführen konnten. Die Auswahl der Erhebungsjahre war dagegen sachlich begründet. Sie ergab sich aus dem Ziel des Projektes, die Rekrutierung der Maires in den unterschiedlichen Staatsformen Frankreichs im 19. und 20. Jahrhundert einerseits und in politischen Ruhelagen andererseits zu untersuchen. ${ }^{8}$ Da die ausgewählten Arrondissements viele
Erhebung darüber hinaus in 50 Städten durch, die im Jahr 1886 als die größsten galten. ${ }^{9}$

1986 veröffentlichte das Team einen Sammelband, in welchem es nicht nur seine Erhebungsmethoden und quantitativen Ergebnisse präsentierte, ${ }^{10}$ sondern zugleich eine Reihe an Aufsätzen veröffentlichte, in denen es um Maires in einzelnen Verwaltungsbezirken (Kommunen, Kantonen, Arrondissements), in >ländlichen oder sstädtischen Regionen`, ferner in der französischsprachigen Literatur seit dem 19. Jahrhundert ging. Mit der Publikation dieser Beiträge verbanden die Herausgeber ein doppeltes Ziel. Sie wollten Perspektiven auf mögliche beziehungsweise notwendige Forschungsfelder eröffnen ${ }^{11}$ und zugleich das von ihnen gewonnene Zahlenmaterial durch qualitative Untersuchungen ergänzen, auf die sie in ihrem Projekt aus pragmatischen Gründen verzichtet hatten. "C'est volontairement«, erklärte Maurice Agulhon, einer der Herausgeber, in der Einleitung des Sammelbandes, »et par simple conscience de la limite des moyens de notre équipe, que nous n'avons pas fait d'efforts de même portée sur des aspects aussi importants de l'histoire des maires que celui de leurs gestions (bien communaux, équipement, assistance, bref budgets), celui de leur installation matérielle (la mairie) et de leur appareil administratif, celui enfin de leurs rapports avec les pouvoirs concurrents ou voisins (le préfet, le clergé, le château, l'usine, la chambre de commerce, les syndicats ...). On en verra cependant mieux que des bribes dans les monographies complémentaires qui vont accompagner l'enquête centrale « ${ }^{12}$ Mit "monographies « meinte er dabei die in dem Band veröffentlichten qualitativen Aufsätze. Der Sammelband war daher weitaus mehr als eine Datensammlung; er bot zugleich Einblicke in die Vielfalt der lokalen Verwaltung und warf Fragen für ihre zukünftige historische Erforschung auf, darunter auch solche, die die Geschichtswissenschaft in der Bundesrepublik gegenwärtig beschäftigen und deshalb gar nicht so neu sind, wie sie aufgrund fehlender Forschungen mitunter erscheinen mögen, etwa die Frage nach der Geschichte von Vermögen und Reichtum im 19. und 20. Jahrhundert, ${ }^{13}$ wie sie vor Kurzem für Deutschland gestellt wurde. ${ }^{14}$

In der zeitgenössischen Forschung erntete die Forschergruppe für ihr Projekt viel Lob und Anerkennung. "L'équipe du Centre de Recherches du XIXe siècle de la Sorbonne [...] présente ici un travail pionnier sur une population, somme toute mal connue: les maires français«, 
rühmte Marc Abélès in seiner 1986 in "Etudes rurales" erschienenen Besprechung des Sammelbandes. ${ }^{15}$ In der Tat hatte das Team mit seiner Studie Beachtliches geleistet. Es hatte historisches Grundlagenwissen über die Rekrutierung der Maires in Frankreich für einen Zeitraum von mehr als 150 Jahren erarbeitet, das bis heute einschlägig geblieben ist. Darüber hinaus hatte es Methoden zur Untersuchung lokaler Eliten entwickelt, die in der französischen Geschichtswissenschaft inzwischen Schule gemacht haben. ${ }^{16}$ Das Verdienst der Forschergruppe ist deshalb nicht hoch genug einzuschätzen. Mit Jocelyne Georges "Histoire des maires de 1789 à 1940 « und André Chandernagors "Les maires en France, $\mathrm{XIX}^{\mathrm{e}}-\mathrm{XX}^{\mathrm{e}}$ siècles" sind inzwischen zwar weitere historische Längsschnittanalysen hinzugetreten. ${ }^{17}$ Obsolet gemacht haben diese Studien den Sammelband indessen nicht.

Es liegt in der Natur der Sache - der Untersuchungszeitraum der Arbeit endet, wie gesagt, in der zweiten Hälfte des 20. Jahrhunderts -, dass das Werk nicht über die Maires der jüngeren Zeitgeschichte informiert. Auch kulturhistorische Fragen lassen sich mit dem Sammelband nur in sehr beschränktem Maße, wenn überhaupt, beantworten. Das gilt etwa für Fragen der Herrschaftsausübung und Staatsbildung, wie die jüngst untersuchte Rolle der Kommunen beim »Vorrücken des Staates in die Fläche« im 19. Jahrhundert. ${ }^{18}$ Es gilt ebenfalls für Fragen nach Kommunikations-, Interaktions- und Aushandlungsprozessen, ${ }^{19}$ ferner für Repräsentationen und Identitätsbildungen. Auf die Konstituierung, Gestaltung und Nutzung von Räumen, die im Zentrum des vorliegenden Themenheftes stehen, gibt der Sammelband auf den ersten Blick ebenfalls keine Antwort, zumal Begriffe wie sespace oder sterritoire`so gut wie gar nicht darin auftauchen. ${ }^{20}$ Doch bietet der Sammelband bei genauerem
Hinsehen sehr wohl Aufschluss über >Raum`, dann nämlich, wenn man aus geschichtstheoretischer Perspektive danach fragt, wie >Raum` in dem Sammelband untersucht und dargestellt wird. Wenn man so vorgeht, entpuppt sich >Raum ‘ erstens als etwas prinzipiell Gegebenes und nicht Gemachtes - sowohl in Form der Kommunen als auch in Gestalt des französischen Staatsgebietes insgesamt. Denn es wurden zwar Veränderungen in der kommunalen Zusammensetzung der 13 untersuchten Arrondissements in die Analyse einbezogen, die Kommunen selbst jedoch als präexistierende, statische Räume quasi vorausgesetzt. Für Frankreich insgesamt blieben die Fluktuationen des Territoriums im 19. Jahrhundert sogar ganz unberücksichtigt, mehr noch: Außerhalb des Hexagons liegende Gebiete in Europa oder Übersee wurden gar nicht in den Sammelband einbezogen, weder in die quantitative Erhebung noch in die qualitativen Untersuchungen, ja, ihre Existenz wurde nicht einmal zur Sprache gebracht. »On regrettera «, erklärten Maurice Agulhon und JeanLouis Robert lediglich in der Darlegung der Erhebungsmethode, "l'absence de certaines régions, particulièrement dans l'extrême-Ouest et le Sud-Ouest «. ${ }^{21}$ >Frankreich` wurde somit nicht nur als eine statische territoriale Entität aufgefasst und untersucht, sondern auch mit >Kontinentalfrankreich gleichgesetzt, für das in Frankreich noch immer `métropole` geläufig ist. Das verweist auf die zweite Bedeutung, die >Raum ‘ in dem Sammelband besaß, und zwar >Raum` als etwas Vorgestelltes, als eine Imagination. Entsprechend ist der Sammelband nicht nur inhaltlich wie methodisch einschlägig für die Sozialgeschichte der Maires in Frankreich bis Mitte des 20. Jahrhundert. Er ist zugleich ein gutes Exempel dafür, wie Raumvorstellungen geschichtswissenschaftliches Arbeiten vorstrukturieren und damit historische Erkenntnisse präjudizieren können. 
"Le maire«, in: Pouvoirs. Revue française d'études constitutionnelles et politiques 24 (1983). A в É L È S, Marc: »M. Agulhon, L. Girard, J.-L. Robert, et al., Les maires en France du Consulat à nos jours«, in: Etudes Rurales 101/102 (1986), S. 311-313. A g U L H O N, Maurice et al.: Les Maires en France du Consulat à nos jours, Paris 1986. • »Introduction«, in: Maurice Agulhon et al.: Les Maires en France du Consulat à nos jours, Paris 1986, S. 7-17. •/ G o S S E Z, Rémi: „Rappel du mode de recrutement«, in: Maurice Agulhon et al.: Les Maires en France du Consulat à nos jours, Paris 1986, S. 28-31. • / G o S S E Z, Rémi / R o B E RT , Jean-Louis: „Les Maires des plus grandes villes«, in: Maurice Agulhon et al.: Les Maires en France du Consulat à nos jours, Paris 1986, S. 139-181. • / R O B E R T, Jean-Louis: "La méthode«, in: Maurice Agulhon et al.: Les Maires en France du Consulat à nos jours, Paris 1986, S. 19-27. • / R O B E R T, Jean-Louis / S E R M A N, William: „Un sondage national: 1600 Maires en 13 arrondissements«, in: Maurice Agulhon et al.: Les Maires en France du Consulat à nos jours, Paris 1986, S. 33-135. A U B ERT, Jacques et al.: Les préfets en France (1800-1940), Genève 1978. B R A K E N S I E K, Stefan / B R E D o W , Corinna von / NÄTHER, Birgit (Hg.): Herrschaft und Verwaltung in der Frühen Neuzeit, Berlin 2014. B R U N E T, Jean-Paul (Hg.): Les conseillers municipaux des villes de France au XXe siècle, Paris 2006. C H A N D E R N A G O R, André: Les maires en France, $X X^{e}-X X^{e}$ siècles. Histoire et sociologie d'une fonction, Paris 1993. G E O R G E, Jocelyne: Histoire des maires de 1789 à 1940, Paris 1989. E I в A C H , Joachim: "Sozialgeschichte«, in: Joachim Eibach / Günther Lottes (Hg.): Kompass der Geschichtswissenschaft. Ein Handbuch, Göttingen 2002, S. 9-22. E s т È B E, Jean: Les Ministres de la République (1871-1914), Paris 1983. F R A N Z, Norbert: »Tätigkeitsfelder und Handlungsspielräume der >letzten Rädchen im Staat`: Durchstaatlichung und Ausweitung der Staatstätigkeit in politisch-administrativen Landgemeinden Frankreichs und Luxemburgs im 19. Jahrhundert«, in: Jörg Ganzenmüller / Tatjana Tönsmeyer (Hg.): Vom Vorrücken des Staates in die Fläche. Ein europäisches Phänomen des langen 19. Jahrhunderts, Köln 2016, S. 111-130. G A J E K, Eva Maria: "Sichtbarmachung von Reichtum. Das Jahrbuch des Vermögens und Einkommens der Millionäre in Preußen«, in: Archiv für Sozialgeschichte 54 (2014), S. 79-108. G I R A R D, Louis / P R OST, Antoine / G OS SEZ, Rémi: Les conseillers généraux en 1870, Paris 1967. • et al.: La Chambre des députés en 1837-1839, Paris 1976. L E B É G u E C, Gilles / P E S C H A N S K I, Denis (Hg.): Les élites locales dans la tourmente. Du Front populaire aux années cinquante, Paris 2000. Le C L ère, Bernard / Wright, Vincent: Les Préfets du Second Empire, Paris 1973. M E S TRE, Jean-Louis: "Le conseil général des Bouches-du-Rhône de 1852 à 1879«, in: Provence historique 84 (1971), S. 177-187. R A U, Susanne: Räume. Konzepte, Wahrnehmungen, Nutzungen, Frankfurt am Main 2013. R o G E R, Patrick: "La France ne compte plus que 35.498 communes«, in: Le Monde (19. Januar 2017), online unter: http://www.lemonde.fr/politique/article/2017/01/19/la-france-necompte-plus-que-35-498-communes_5065260_823448.html (17. 6. 2017). S ü s s, Winfried: „Gold ist Trumpf und weiter nichts. Reichtumskonflikte im langen 19. Jahrhundert«, in: WerkstattGeschichte 73 (2017), S. 31-49. T I м в A L, Pierre-Clément / C A S TA L D O, André: Histoire des institutions publiques et des faits sociaux, Paris 1990. T U DES Q, André-Jean: Les conseillers généraux en France au temps de Guizot 1840-1848, Paris 1967. 
1 Patrick Roger: "La France ne compte plus que 35.498 communes«, in: Le Monde (19. Januar 2017), online unter: http://www.lemonde. $\mathrm{fr} /$ politique/article/2017/01/19/la-france-ne-compte-plus-que35-498-communes_5065260_823448.html (17.6. 2017).

2 Eine Zusammenschau dieser Reformen bieten Pierre-Clément Timbal / André Castaldo: Histoire des institutions publiques et des faits sociaux, Paris 1990, S. 680-686; ferner vgl. André Chandernagor: Les maires en France, XIXe $-X X^{e}$ siècles. Histoire et sociologie d'une fonction, Paris 1993, S. 17-18.

3 Zu den Veränderungen der Rekrutierung vgl. Maurice Agulhon / Rémi Gossez: »Rappel du mode de recrutement«, in: Maurice Agulhon et al.: Les Maires en France du Consulat à nos jours, Paris 1986, S. 28-31.

4 Maurice Agulhon: "Introduction«, in: Maurice Agulhon et al.: Les Maires en France du Consulat à nos jours, Paris 1986, S. 7-17, hier S. 8; ferner vgl. Chandernagor: Maires, S. 33-34; Norbert Franz: "Tätigkeitsfelder und Handlungsspielräume der ıletzten Rädchen im Staatı: Durchstaatlichung und Ausweitung der Staatstätigkeit in politisch-administrativen Landgemeinden Frankreichs und Luxemburgs im 19. Jahrhundert«, in: Jörg Ganzenmüller / Tatjana Tönsmeyer (Hg.): Vom Vorrücken des Staates in die Fläche. Ein europäisches Phänomen des langen 19. Jahrhunderts, Köln 2016, S. 111-130, hier S. 114.

5 Siehe den historiografischen Überblick von Joachim Eibach, "Sozialgeschichte«, in: Joachim Eibach / Günther Lottes (Hg.): Kompass der Geschichtswissenschaft. Ein Handbuch, Göttingen 2002, S. 9-22, hier S. 19. In Frankreich entstanden in diesem Zusammenhang unter anderem Studien über unterschiedliche Gruppen von Verwaltungsbeamten und Abgeordneten; André-Jean Tudesq: Les conseillers généraux en France au temps de Guizot 1840-1848, Paris 1967; JeanLouis Mestre: "Le conseil général des Bouches-du-Rhône de 1852 à 1879«, in: Provence historique 84 (1971), S. 177-187; Jacques Aubert et al.: Les préfets en France (1800-1940), Genève 1978; Bernard Le Clère / Vincent Wright: Les Préfets du Second Empire, Paris 1973; Jean Estèbe: Les Ministres de la République (1871-1914), Paris 1983.

6 Siehe etwa die Beiträge im Zeitschriftenheft "Le maire«, in: Pouvoirs. Revue française d'études constitutionnelles et politiques 24 (1983).

7 Louis Girard / Antoine Prost / Rémi Gossez: Les conseillers généraux en 1870, Paris 1967; Louis Girard et al.: La Chambre des députés en 1837-1839, Paris 1976.

8 Maurice Agulhon / Jean-Louis Robert: "La méthode«, in: Maurice Agulhon et al.: Les Maires en France du Consulat à nos jours, Paris 1986, S. 19-27, hier S. 20-21.

9 Maurice Agulhon / Rémi Gossez / Jean-Louis Robert: »Les Maires des plus grandes villes«, in: Maurice Agulhon et al.: Les Maires en France du Consulat à nos jours, Paris 1986, S. 139-181, hier S. 139.

10 Maurice Agulhon / Jean-Louis Robert / Willliam Serman: „Un sondage national: 1600 Maires en 13 arrondissements", in: Maurice Agulhon et al.: Les Maires en France du Consulat à nos jours, Paris 1986, S. 33-135.

11 Agulhon / Robert / Serman: "Sondage«, S. 55.

12 Agulhon: »Introduction«, S. 16-17.

13 Agulhon / Robert / Serman: "Sondage«, S. 36

14 Eva Maria Gajek, "Sichtbarmachung von Reichtum. Das Jahrbuch des Vermögens und Einkommens der Millionäre in Preußen«, in: Archiv für Sozialgeschichte 54 (2014), S. 79-108; Winfried Süß: „Gold ist Trumpf und weiter nichts. Reichtumskonflikte im langen 19. Jahrhundert«, in: WerkstattGeschichte 73 (2017), S. 31-49.

15 Marc Abélès: »M. Agulhon, L. Girard, J.-L. Robert, et al., Les maires en France du Consulat à nos jours«, in: Etudes rurales 101/102 (1986), S. 311-313, hier S. 311.

16 Etwa bei der Untersuchung der Munizipalräte französischer Städte im 20. Jahrhundert, deren Ergebnisse in folgendem Sammelband veröffentlicht wurden: Jean-Paul Brunet (Hg.): Les conseillers municipaux des villes de France au XXe siècle, Paris 2006.

17 Jocelyne George: Histoire des maires de 1789 à 1940, Paris 1989; Chandernagor: Maires. Erwähnenswert ist ferner folgender Sammelband: Gilles Le Béguec / Denis Peschanski (Hg.): Les élites locales dans la tourmente. Du Front populaire aux années cinquante, Paris 2000.

18 Jörg Ganzenmüller / Tatjana Tönsmeyer (Hg.): Vom Vorrücken des Staates in die Fläche. Ein europäisches Phänomen des langen 19. Jahrhunderts, Köln 2016.

19 Stefan Brakensiek / Corinna von Bredow / Birgit Näther (Hg.): Herrschaft und Verwaltung in der Frühen Neuzeit, Berlin 2014.

20 Zu Raumkonzepten der französischsprachigen Forschung vgl. Susanne Rau: Räume. Konzepte, Wahrnehmungen, Nutzungen, Frankfurt am Main 2013, S. 39-52.

21 Agulhon / Robert: »Méthode«, S. 20. 


\section{Abstract}

The volume "Les Maires en France du Consulat à nos jours«, published in France in 1986, was the first historical work to open long-term perspectives on French mayors in the 19th and 20th century. On the one hand, these perspectives resulted from the data obtained within the framework of a quantitative long-term analysis; on the other hand, they relied on qualitative explorations of selected administrative units or regions. In re-reading "Les Maires en France du Consulat à nos jours", this article shows that the volume has remained a reference work for the history of French municipalities until today, even though it does not always allow answering current research questions.

\section{About the Author}

Bettina Severin-Barboutie is Professor of History at Giessen University and member of different research groups working on Europe, among them the research cluster »Writing a New History of Europe« at the University Paris I Panthéon-Sorbonne. Her work focuses on state and empire building, mobility and migration, visual culture and the transmission of history in and outside of Europe from the 18th to the 21st century. She is the author of »Französische Herrschaftspolitik und Modernisierung. Verfassungs- und Verwaltungsreformen im Großherzogtum Berg (1806-1813)«, editor of »Stadt in Bewegung. Wanderungsprozesse in pluridisziplinärer Perspektive« (2014), and co-editor of "Verflochtene Vergangenheiten. Geschichtscomics in Europa, Asien und Amerika. Perspektiven auf ein Forschungsfeld" (2014). She is currently writing a manuscript on mobility and migration in the cities of Stuttgart and Lyon after 1945. 